\title{
CAD-Assisted Microwave Characterization of Ink-Jet Printed CPW on PET Substrates
}

\author{
A. Sahu*,V. Devabhaktuni*,A. Lewandowski ${ }^{\dagger}$, T. M. Wallis ${ }^{\ddagger}$, and P. H. Aaen ${ }^{\S}$ \\ *Dept. of Electrical Engineering and Computer Science,The University of Toledo, Toledo, Ohio, USA \\ $\dagger$ Institute of Electronic Systems, Warsaw University of Technology, Warsaw, Poland \\ ${ }^{\ddagger}$ National Institute of Standards and Technology Boulder, Colorado, USA

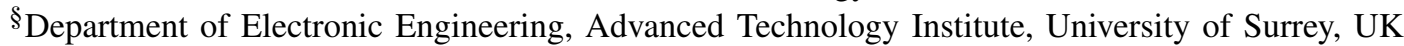 \\ Email: Abhishek.Sahu@utoledo.edu
}

\begin{abstract}
This paper describes the computer-aided-design (CAD)-assited microwave characterization of coplanar waveguide (CPW) lines formed by ink-jet printed technology on flexible polyethylene terephthalate (PET) substrates. A spacemapping technique is used to link the measured results with simulations and the mapping is used to extract the electrical properties of the ink and supporting dielectric spacer employed during measurements. Results indicate that the losses in lines are predominantly due to the loss tangent of the substrate and the conductivity of the ink. Estimates of the conductivity of the ink and the dielectric constant of the spacer used during measurement were obtained as $2.97 \times 10^{7} \mathrm{~S} / \mathrm{m}$, and 1.79 respectively.
\end{abstract}

Index Terms - Ink-jet printed CPW, flexible substrates, multiline calibration, CAD

\section{INTRODUCTION}

To enable microwave circuits for emerging markets like the internet-of-things (IoT), there is a need for the inexpensive manufacture of environmentally friendly electronics and ink-jet printing has received significant recent interest. Inkjet printing allows the use of non-traditional substrate materials, for example polyethylene terephthalate (PET) and paper, which are very inexpensive. In addition, these substrates are mechanically flexible and can conform to non-planar geometries. The manufacturing process is additive and only the ink needs to be added to the substrate. Circuits can then be printed using ink-jet printers or even roll-to-roll printing systems. Additive manufacturing is suitable for rapid, costeffective mass manufacturing. Recent research has led to the development of various components such as radio frequency identification (RFID) tags [1], antennas [2], [3], and coplanar waveguides (CPW) [4] by use of ink-jet printing on flexible substrates such as Kapton [5].

Polyethylene terephthalate (PET) is a widely used flexible substrate that is being explored for microwave circuit design. CPWs on flexible PET were reported to have an attenuation of $0.6 \mathrm{~dB} / \mathrm{mm}$ at $40 \mathrm{GHz}$ [6]. Furthermore, meander inductors have been successfully designed on PET substrates [7], [8]. In order to understand the limitations of substrate selection and to offer development guidance on new, nanoparticle based metallic inks and their processing, there is a need to have robust, repeatable and accurate characterization.

Recently, the authors presented calibrated microwave measurements of ink-jet-printed CPWs on a PET substrate as

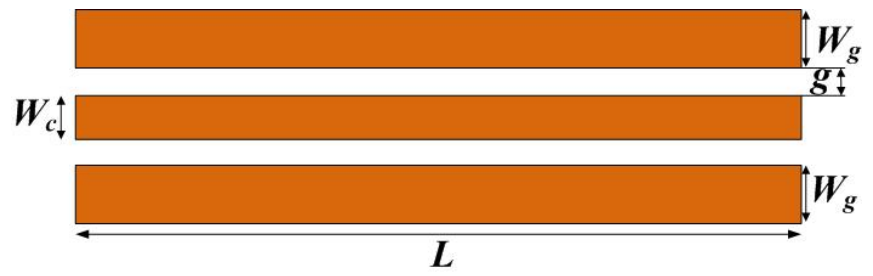

(a)

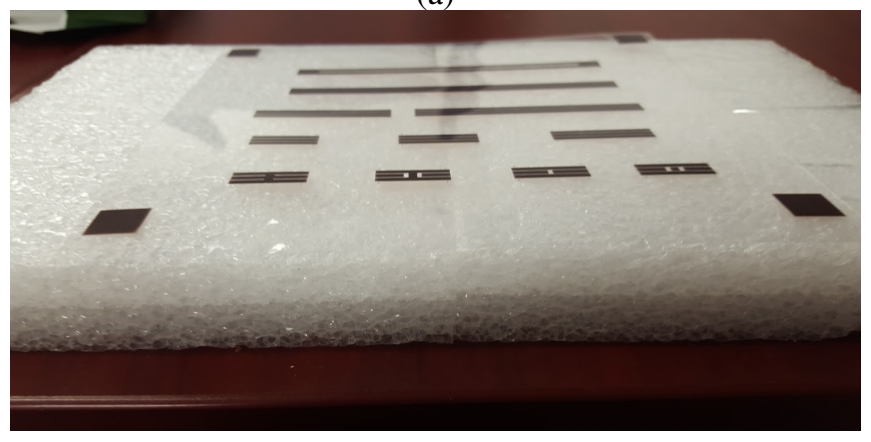

(b)

Fig. 1. (a) CPW dimensions (b) Photograph of fabricated CPW lines on PET substrates with spacer underneath.

a first step toward developing a broadly-applicable test platform for evaluation of flexible, ink-jet-printed microwave circuits as well as their constituent materials [9]. Utlizing a novel metrology, it was shown that repeatable measurements can be performed for inkjet printed circuits on PET. Nevertheless, there is an urgent need for a robust metrology to quantitatively characterize the constituent inks and components fabricated using such techniques.

A TDR/TRT and S-parameter measurement based characterization of ink-jet printed interconnects was reported in [10]. Electrical performance characterization of a flexible circuit for mobile application was presented in [11]. Nevertheless, a robust metrology to characterize the ink properties on PET-based substrates is yet to be reported. To this end, we report a CAD-assisted space mapping technique to characterize ink-jet-printed CPW lines on a PET substrate. Section II describes the fabrication and measurement of CPW lines. Characterization and parameter extraction methodology is described in section III. Results and conclusions are discussed in section IV and V. 


\section{FABRiCATION AND MEASUREMENT OF CPW Lines}

\section{A. Fabrication}

The fabrication process was performed using a commercial process [12]. The process uses ink-jet printing as a precursor on which copper is electroplated. In this work, the copper is plated to be approximately $2 \mu \mathrm{m}$ thick. We used a clear PET substrate for our work due to its much lower cost compared to other flexible circuits such as polymide and Kapton, high chemical resistance to acids and solvents, and ability to withstand higher operating temperature than other thermoplastics. Contact profilometer measurements were performed on the traces revealed their thickness to be approximately $2 \mu \mathrm{m}$ over the entire substrate with a surface roughness of about $0.2 \mu \mathrm{m}$.

Figure 1(a) is a photograph of a CPW line with $\left(W_{c}\right)$ $1.983 \mathrm{~mm}$, gap width $(g) 0.13 \mathrm{~mm}$, ground conductor width $\left(W_{g}\right) 1.983 \mathrm{~mm}$, substrate thickness $125 \mu \mathrm{m}$ and copper thickness $2 \mu \mathrm{m}$. The line lengths were determined based on the approach in reference [13]. A multiline TRL calibration kit was designed with six CPW lines having lengths of 14.97 $\mathrm{mm}, 18.42 \mathrm{~mm}, 23.57 \mathrm{~mm}, 35.78 \mathrm{~mm}, 61.60 \mathrm{~mm}$, and 97.93 $\mathrm{mm}$. Additionally, a CPW Short circuit has been fabricated for use as a reflect standard in the mulitline TRL calibration.

\section{B. Measurement}

The CPW lines were measured using a commercial onwafer probe station, a vector network analyzer (VNA), and ground-signal-ground contact probes $(1.0 \mathrm{~mm}$ pitch). During measurements, the PET substrate was supported by a 0.5 $\mathrm{cm}$-thick, porous dielectric. The power level of the VNA was set to $-17.0 \mathrm{dBm}$ and the uncorrected S-parameters were measured for each device from $0.1 \mathrm{GHz}$ to $20 \mathrm{GHz}$. We adopted a multiline Thru-Reflect-Line calibration [6] technique to characterize the propagation constant and reflect reflection coefficient of the CPW lines.

\section{Characterization AND PARAMETER EXTRACTION}

We utilized a CAD-based space mapping methodology to characterize the fabricated ink-jet printed CPW lines. To this end, the dielectric constant and loss tangent of PET are well characterized in literature and hence were kept as their standard values as 3.1 and 0.01 respectively. A simulation was performed utilizing these values to compare with measurement. CPW lines were drawn on PET substrates and the dielectric spacer was then designed underneath. Open boundary conditions were defined around the model and at the bottom PEC boundary condition was defined as per the measurement set up. Figure 2 shows the simulation setup developed in Ansys's HFSS ${ }^{\|}$to carry out the EM simulation.

Since there are two unknown parameters in the experiment, let us define the conductivity of the copper electroplated ink-jet printed lines as sigma and the dielectric constant of the spacer underneath as epsr. The resulting attenuation and phase constants here are defined as $\alpha$ and $\beta$.

\footnotetext{
" Trade names are used for clarity and do not imply endorsement by NIST.
}

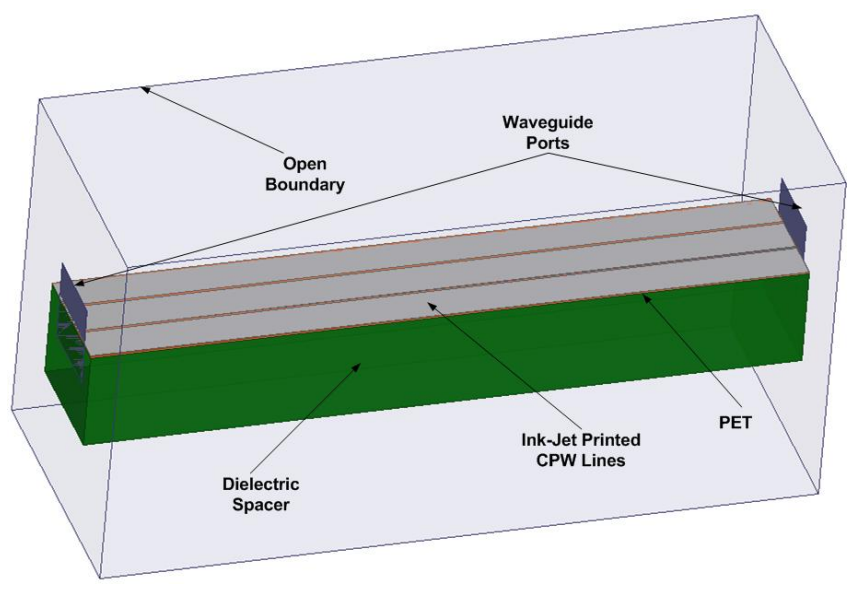

Fig. 2. Simulation setup of CPW lines for proposed methodology.

The proposed methodology to extract the unknown parameters from simulation and measurement data set is summarized as below.

step 1: Start the simulation with an initial value of sigma $_{0}$ and $\overline{e p s} r_{0}$.

step 2: Keep the sigma $_{0}$ fixed and find different values of $\bar{\beta}$ by varying epsr 0 . Define an optimization function as the difference of the measurement and the simulated results. Obtain an optimized value of epsr 0 to provide the best match for beta between EM simulation and measurement data set. step 3: Following step 2, obtain a sigma that provides the best match for $\alpha$ between Em simulation and measurement data set. Define this as sigma .

step 4: keep sigma ${ }_{1}$ fixed, and obtain epsr 1 to give best $\overline{\text { match }}$ for $\beta$ between simulation and measurement.

step $n$ : Iterate step 1- step 3 till you get a convergence between simulation and measurement grids for sigma and epsr.

The above approach is easily cast in the framework of space mapping [14]. In the above context, the measurement data set is the fine model and the EM analysis result is the coarse model.

\section{RESUlts AND ANALYSIS}

Following the methodology described above, we performed several iterations to obtain the value of unknown parameters. The dielectric constant and loss tangent of PET substrate were defined as 3.1 and 0.01 . Additionally, the initial values for sigma and epsr were set as $8.54 \times 10^{7} \mathrm{~S} / \mathrm{m}$ (same as copper) and 3.1 (same as PET) to define an uniform model for iteration 1. After each iteration, we updated the search range by observing the results from previous iterations. It was observed that the search engine converged after six iterations. The final values of sigma of the ink and epsr of the dielectric spacer were found to be $2.97 \times 10^{7} \mathrm{~S} / \mathrm{m}$ and 1.79. Figure 3 depicts the comparison of attenuation and phase constant plots from EM simulation and measurement. As can be observed, both the results are in agreement, which demonstrates the general effectiveness of the proposed methodology. However, there is a slight mismatch for the 


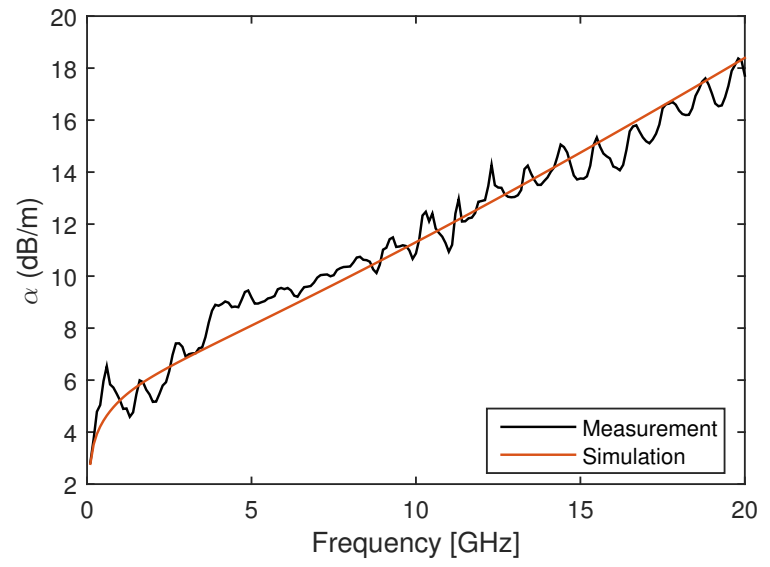

(a)

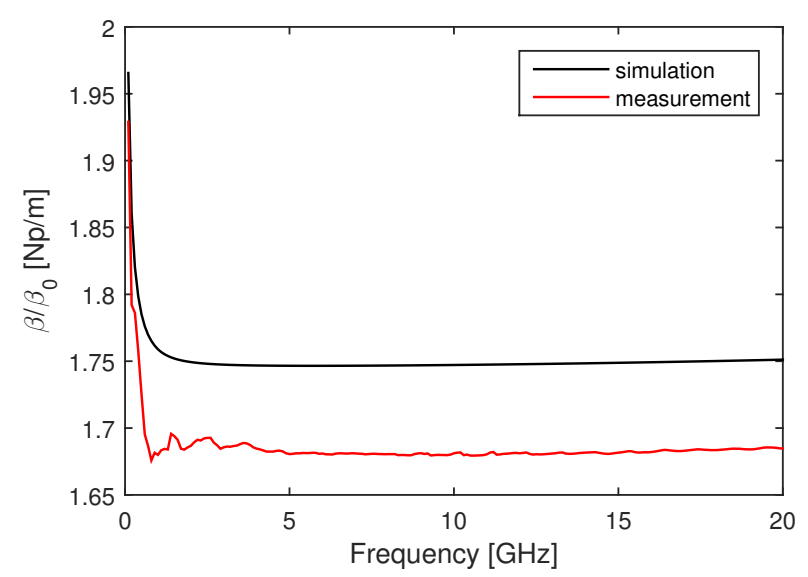

(b)

Fig. 3. (a) Comparison of attenuation constant from measurement and simulation (b) Comparison of normalized phase constant from measurement and simulation

normalized $\beta$ from simulation and measurement which is attributed to the small gap between the porous dielectric and PET. The ripples in the measured attenuation might be due to imprecise probe positioning during measurement. Furthermore, it was also observed that the losses in the CPW lines are mostly affected by the conductivity of the ink and loss tangent of PET substrate. The loss tangent of the dielectric spacer has minimal effect on the overall losses.

\section{CONCLUSIONS}

This paper proposes a methodology for characterization of CPW lines on flexible PET substrate. Using a CADbased space mapping method, we were able to extract the conductivity of the electroplated copper ink and the dielectric constant of the spacer used in the measurement. By utilizing the known parameters of PET from fine model, the unknown ink conductivity and spacer dielectric constant are extracted from the coarse model as $2.9734 \times 10^{7} \mathrm{~S} / \mathrm{m}$ and 1.786 respectively. Results obtained from simulation agree quite well with measurement. However, as the porous dielectric is not entirely flat there is a small gap between the PET and the spacer that leads to a slight mismatch in results. Together these results demonstrate a viable and robust technique for characterization of ink-jet printed microwave circuits as well as the constituent materials.

\section{REFERENCES}

[1] M. A. Ziai and J. C. Batchelor, "Temporary on-skin passive UHF RFID transfer tag," IEEE Trans. Antennas Propag., vol. 59, no. 10, pp. 35653571, Oct. 2011.

[2] G.A. Casula, G. Montisci and G. Mazzarella, "A Wideband PET InkjetPrinted Antenna for UHF RFID," IEEE Antennas Wireless Propag. Lett., vol. 12, pp. 1400-1403, 2013.

[3] T. Peter, Y.Y. Sun, T.I. Yuk, H.F. AbuTarboush, R. Nilavalan and S.W.Cheung, "Miniature transparent UWB antenna with tunable notch for green wireless applications," Antenna Technology (iWAT), 2011 International Workshop on, pp. 259-262.

[4] K. Hettak, T. Ross, R. James, A. Momciu and J. Wight, "Flexible Polyethylene Terephthalate-based inkjet printed CPW-fed monopole antenna for $60 \mathrm{GHz}$ ISM applications," Microwave Conference (EuMC), 2013 European, pp. 1447-1450.

[5] S. Swaisaenyakorn, P. R. Young and M. Shkunov,"Characterization of inl-jet printed CPW on Kapton substrates at $60 \mathrm{GHz}$ " Loughborough Antennas and Propagation Conference (LAPC), 2014, UK, pp. 676678.

[6] K. Hettak et al, "Low-cost mm-wave coplanar waveguide bandpass filter using inkjet printing of silver nano-particles on flexible plastic substrate," Proc. IEEE International Microwave Symposium, 17-22 May 2015, pp: 1-3.

[7] A. B. Menicanin, L. D. Zivanov, M. S. Damnjanovic and A. M. Maric, "Low-cost CPW meander inductors utilizing ink-jet printing on flexible substrate for high-frequency applications," IEEE Trans. Electron Devices, vol. 60, no. 2, pp. 827-832, Jan. 2013.

[8] A. Manicanin, L. Zivanov, C. Zlebic, M. Kisic, N. Blaz and M. damnzanovic, "Fully inkjet printed CPW meander inductors on PET flexible substrate," in 38th Int. Spring Seminar on Elect. Tech., Eger, May 6-10 2015, pp. 43-46.

[9] A. Sahu, V. Devabhaktuni, A. Lewandowski, T. M. Wallis, and P. Aaen, "Microwave Characterization of Ink-Jet Printed CPW on PET Substrates," in 86th ARFTG Microwav eMeasurment conference., Atlanta, GA, Dec 3-4 2015, pp. 1-4.

[10] B. Shao, R. Weerasekara, A. T. Woldegiorgis, Li-Rong Zheng, R. Liu, and W. Zapka, "High frequency characterization and modelling of inkjet printed interconnects on flexible substrate for low-cost RFID applications," Electronics System-Integration Technology Conference, Greenwich, 1-4 Sept. 2008, pp. 695-700.

[11] S. Koskinen, L. Pykari, and M. Mantisalo, "Electrical Performance Characterization of an Inkjet-Printed Flexible Circuit in a Mobile Applications," IEEE Trans.Compon. Packag. Manufac. Tech., vol. 3, no. 9, pp. 1604-1610, Sep. 2013.

[12] http://inkjetflex.com/site/rapid-prototyping/.

[13] A. Lewandowski, W. Wiatr, L.J. Opalski, and R. Biedrzycki, "Accuracy and bandwidth optimization of the over-determined offset-short reflectometer calibration" IEEE Trans. Microw. Theory Tech., vol. 63, no. 3, pp. 1076-1089, March 2015.

[14] J. C. Rautio, and S. Arvas, "Measurement of Planar Substrate Uniaxial Anisotropy" IEEE Trans. Microw. Theory Tech., vol. 57, no. 10, pp. 2456-2463, Oct. 2009. 